\title{
Generation of Variable-Length Time Series from Text using Dynamic Time Warping-Based Method
}

\author{
Ayaka Ideno \\ ideno@mi.t.u-tokyo.ac.jp \\ The University of Tokyo \\ Meguro, Tokyo, Japan
}

\author{
Yusuke Mukuta \\ mukuta@mi.t.u-tokyo.ac.jp \\ The University of Tokyo \\ Meguro, Tokyo, Japan \\ RIKEN \\ Chuo, Tokyo, Japan
}

\author{
Tatsuya Harada \\ harada@mi.t.u-tokyo.ac.jp \\ The University of Tokyo \\ Meguro, Tokyo, Japan \\ RIKEN \\ Chuo, Tokyo, Japan
}

\begin{abstract}
This study is aimed at finding a suitable method for generating timeseries data such as video clips or avatar motions from text stating multiple events. This paper addresses the generation of variablelength time-series data considering the order and variable duration of events stated in the text. Although the use of the variant of Mean Squared Error (MSE) is a common means of training, only the gap between the element of ground-truth (GT) data and generated data at the same time are considered. Thus, variants of MSE are unsuitable for the task at hand because the loss may not be small for the generated and GT data with the same order of events if the time for each event does not overlap. To solve the problem, we propose a Dynamic Time Warping-Like method for Variable-Length data (DTWL-VL), which determines the corresponding elements of the GT and the generated data, allowing for the time difference between them, and makes them closer. We compared DTWL-VL, a variant of MSE, and an existing method for time-series data generation which considers the time difference between the corresponding part in the GT and generated data. Since the existing method is aimed at generating fixed-length data, we extend the method for generating variable-length time-series data. We conducted experiments using a dataset prepared for this study. Both DTWL-VL and the existing methods outperformed the MSE variant. Moreover, although the existing method outperformed DTWL-VL under certain settings, DTWL-VL required a smaller training period.
\end{abstract}

\section{CCS CONCEPTS}

- Computing methodologies $\rightarrow$ Neural networks; Structured outputs; Information extraction.

\section{KEYWORDS}

time-series generation, text to time-series data, time-series matching, text processing, variable length

ACM Reference Format:

Ayaka Ideno, Yusuke Mukuta, and Tatsuya Harada. 2021. Generation of Variable-Length Time Series from Text using Dynamic Time Warping-Based

Permission to make digital or hard copies of all or part of this work for personal or classroom use is granted without fee provided that copies are not made or distributed for profit or commercial advantage and that copies bear this notice and the full citation on the first page. Copyrights for components of this work owned by others than the author(s) must be honored. Abstracting with credit is permitted. To copy otherwise, or republish, to post on servers or to redistribute to lists, requires prior specific permission and/or a fee. Request permissions from permissions@acm.org.

MMAsia '21, December 1-3, 2021, Gold Coast, Australia

(c) 2021 Copyright held by the owner/author(s). Publication rights licensed to ACM. ACM ISBN 978-1-4503-8607-4/21/12 ..\$15.00

https://doi.org/10.1145/3469877.3495644
Method. In ACM Multimedia Asia (MMAsia '21), December 1-3, 2021, Gold Coast, Australia. ACM, New York, NY, USA, 7 pages. https://doi.org/10.1145/ 3469877.3495644

\section{INTRODUCTION}

Time-series data, such as video sequences and avatar motions, are extensively used. Automatic generation of such data from text will reduce creation costs because text creation does not require any special technique or equipment. Although existing studies have addressed this problem $[1-3,5,9,14-19,21,24,25]$, they have not focused on reflecting the appropriate order and duration of sequential events. For example, to the best of our knowledge, no studies have attempted to generate video sequences from text including multiple sequential actions such as "A man was walking. He suddenly stopped and picked something up." When the input text involves multiple events, the generated time-series data should have an appropriate order of events to reflect the meaning of the input text. Furthermore, the duration of each event should be appropriate. It means that the duration of events in the output data should not deviate significantly from the distribution of the duration in groundtruth (GT) data. Moreover, since the length of the time-series data may be affected by the content of the input text, the generation of variable-length time-series data is required. It is therefore necessary to develop a method for the generation of time-series data with the proper order and natural duration of events, and variable length.

Loss functions like Mean Squared Error (MSE) have been used in many studies. However, MSE may be unsuitable for generating data with proper order of events. This is because MSE does not align elements with different time, and data with the same order of events are not regarded as similar if the time of events in the two data do not overlap. Therefore, to generate data with the appropriate order and natural duration of events, we propose a method that can align elements of time-series data at different time.

Our contributions are summarized as follows:

- To generate time-series data with proper order and natural duration of events, we propose DTWL-VL, which considers the alignment between time-series data, and causes the duration and value of the corresponding parts to be closer.

- We built a synthetic dataset that consists of a set of text that states the sequential action of people and corresponding variable-length time-series data representing the action of such people at all times.

- In our experiments, we compared DTWL-VL to an existing method that originally considers the alignment between time-series data with the same length. We demonstrated that 
incorporating the loss of the inactive part of the data for generating variable-length data improves the performance of the existing method. Furthermore, both DTWL-VL and the existing method outperform a variant of MSE. Although the existing method outperforms DTWL-VL under certain settings, DTWL-VL requires a shorter training period.

\section{RELATED WORK}

\subsection{Generation of time-series data from text}

Several studies have addressed time-series data generation from text. $[1,15,21,24,25]$ tackled motion generation, and $[2,3,5,9,14,16-$ 19] focused on video generation. However, none of these studies focused on reflecting multiple sequential events in the output or considering the time difference between the corresponding part of the GT and generated data. Data alignment has been used in speech generation [10]. However, the methods of the previous research are based on the assumption that the alignment between speech and text is monotonic, that is, skipping or repetition of the text input does not occur. This is not the case with video or motion generation because it is not guaranteed that the events are stated in the order they occur. Moreover, the above studies on the generation of video and motion did not investigate a variable length output except [21]

\subsection{Measure for time-series data similarity}

Dynamic Time Warping (DTW)[6] is one of the means to calculate the similarity between time-series data of different lengths. DTW determines the minimal alignment cost from all alignments between the two time-series data. Thus, DTW allows an element in one data to be aligned with an element in the other data at a different time. We denote the DTW between the time-series data $A_{1}^{p}$, the time-series data $A$ from an element with index 1 to $p$, and $B_{1}^{q}$, the time-series data $B$ from the element with index 1 to $q$, as $\delta\left(A_{1}^{p}, B_{1}^{q}\right)$. We call the element of $A_{1}^{p}$ with index $p, a_{p}$, and the element of $B_{1}^{q}$ with index $q, b_{q} . \delta\left(A_{1}^{p}, B_{1}^{q}\right)$ is calculated as follows:

$$
\delta\left(A_{1}^{p}, B_{1}^{q}\right)=\min \left\{\begin{array}{l}
\delta\left(A_{1}^{p-1}, B_{1}^{q}\right)+d\left(a_{p}, b_{q}\right) \\
\delta\left(A_{1}^{p-1}, B_{1}^{q-1}\right)+d\left(a_{p}, b_{q}\right), \\
\delta\left(A_{1}^{p}, B_{1}^{q-1}\right)+d\left(a_{p}, b_{q}\right)
\end{array},\right.
$$

where $\delta$ are initialized as

$$
\begin{array}{r}
\delta\left(A_{1}^{1}, B_{1}^{1}\right)=d\left(a^{1}, b^{1}\right), \\
\delta\left(A_{1}^{p}, B_{1}^{0}\right)=\infty(\text { if } p>0), \\
\delta\left(A_{1}^{0}, B_{1}^{q}\right)=\infty(\text { if } q>0)
\end{array}
$$

, in which $d\left(a^{\prime}, b^{\prime}\right)$ is the distance set between $a^{\prime}$ and $b^{\prime}$.

Figure 1 presents two examples of alignment.

\subsection{Objective for time-series data dissimilarity}

Several existing studies have proposed a differentiable function based on DTW for use as a loss function. Soft-DTW [4] is the softminimum of all alignment costs, whereas DILATE[12] is a combination of soft-DTW and additional loss for time difference between aligned elements. These loss functions can be used to generate time-series data considering time difference of the corresponding part of the GT and generated data. In these studies, the length of the

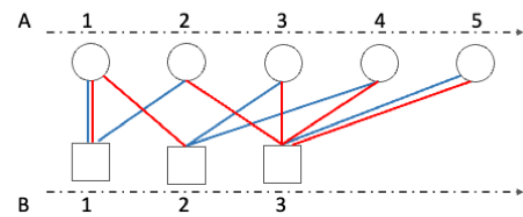

Figure 1: Example of alignment: The cost of alignment is the sum of the distances between aligned elements.The red and blue paths represent examples of alignments. The alignment cost for the red path is $d(A 1, B 1)+d(A 1, B 2)+d(A 2, B 3)+$ $d(A 3, B 3)+d(A 4, B 3)+d(A 5, B 3)$,

generated data was determined, and the methods were not applied to the generation of variable-length time-series data.

\section{PROPOSED MATCHING LOSS}

We propose DTWL-VL to deal with the time difference between the corresponding parts of GT and generated time-series data and generate variable-length outputs. Furthermore, we extend the existing DILATE method to variable-length outputs. We describe the details of DTWL-VL and DILATE-based methods in Sections 3.1 and 3.2, respectively. We consider that elements corresponding to the same event have similar values, and our goal is to generate time-series data with the appropriate order and duration of events described in the input text. Both methods require the model to generate not only the features of the output but also the end possibility for each time in order to generate variable-length time-series data.

\subsection{DTWL-VL}

This method consists of two phases. The first phase is aimed at learning the alignment between the GT and the generated data. In the second phase, the model makes elements that should be aligned closer, based on the alignment obtained during the previous phase.

3.1.1 Components of loss for first phase. Let $A$ be the output data with maxpos elements and $B_{1}^{S}$ be the GT data with $S$ elements. The proposed loss function for the first part is as follows:

$$
\min _{r}(A L(r)+E P(r)+E G(r)),
$$

where $r$ is an index of $A$. The loss corresponds to a certain $r$ with a minimal value, and we refer to this index as $R$.

$A L(r)$ is the loss for the alignment aimed at making the generated time-series data closer to the GT data. We will describe $A L(r)$ later. $E P(r)$ is the penalty for the end position and

$$
E P(r)=\gamma \cdot \mid r-\text { endpos } \mid,
$$

where $\gamma$ is a hyperparameter set to 3.2 in this research, and endpos is the first index with an end possibility of more than 0.5 if any, and the index with the highest probability otherwise. By including the penalty, we discourage the model from determining $R$ ignoring the calculated end position of the generated data, which we expect to help the model learn the appropriate end position.

$E G(r)$ makes the end possibility of $a_{R+1}$ closer to 1 . This is for modifying duration of the part whose end possibility is no more than 0.5 in the same manner to other events as described below.

$$
E G(r)=\lambda \cdot s v\left(\log \left(p_{r+1}^{e n d}\right)\right)
$$


$\lambda$ is a hyperparameter set to 0.1 . This $\lambda$ is the same to $\lambda$ in Eqn. 19 We represent the end possibility of an element at time i as $p_{i}^{\text {end }}$ We define $s g(\cdot)$ to return a value that is equal to its argument but detached from its gradient information. Moreover, we define $s v(\cdot)$ as $s v(A)=A-s g(A)$. The return value of $s v(\cdot)$ is equal to zero.

3.1.2 Details of loss for time-series data alignment. $A L(r)$ is set to $\delta_{\lambda, \text { match }}\left(A_{1}^{r}, B_{1}^{S}\right), A_{1}^{r}$ is the output data with $r$ elements, and $B_{1}^{S}$ is the GT data with $S$ elements. In addition, $\delta_{\lambda \text {,match }}\left(A_{1}^{r}, B_{1}^{S}\right)$ is the matching loss between $A_{1}^{r}$ and $B_{1}^{S}$, which is calculated as

$$
\begin{aligned}
& \delta_{\lambda, \operatorname{match}}\left(A_{1}^{r}, B_{1}^{s}\right)=d\left(a_{r}, b_{s}\right)+\lambda|d n|+g(r, s) \\
& +m c+\min \left\{\begin{array}{l}
\delta_{\lambda, \operatorname{match}}\left(A_{1}^{r-1}, B_{1}^{s-1}\right) \\
\delta_{\lambda, \operatorname{del} A}\left(A_{1}^{r-1}, B_{1}^{s-1}\right) \\
\delta_{\lambda, \operatorname{del} B}\left(A_{1}^{r-1}, B_{1}^{s-1}\right)
\end{array},\right. \\
& \delta_{\lambda, \operatorname{del} A}\left(A_{1}^{r}, B_{1}^{s}\right)=d\left(a_{r}, b_{s}\right)+\min \left\{\begin{array}{l}
\delta_{\lambda, \operatorname{match}}\left(A_{1}^{r-1}, B_{1}^{s}\right) \\
\delta_{\lambda, \operatorname{del} A}\left(A_{1}^{r-1}, B_{1}^{s}\right)
\end{array},\right. \\
& \delta_{\lambda, \operatorname{del} B}\left(A_{1}^{r}, B_{1}^{s}\right)=\operatorname{sg}\left(d\left(a_{r}, b_{s}\right)\right)+\min \left\{\begin{array}{l}
\delta_{\lambda, \operatorname{match}}\left(A_{1}^{r}, B_{1}^{s-1}\right) \\
\delta_{\lambda, \operatorname{del} B}\left(A_{1}^{r}, B_{1}^{s-1}\right)
\end{array},\right.
\end{aligned}
$$

where $\delta$ are defined as

$$
\begin{aligned}
\delta_{\lambda, \text { match }}\left(A_{1}^{1}, B_{1}^{1}\right) & =d\left(a^{1}, b^{1}\right) \\
\delta_{\lambda, \text { match,delA,delB }}\left(A_{1}^{r}, B_{1}^{0}\right) & =\infty(\text { if } r>=0) \\
\delta_{\lambda, \text { match,delA,delB }}\left(A_{1}^{0}, B_{1}^{S}\right) & =\infty(\text { if } s>=0) \\
\delta_{\lambda, \operatorname{del} B}\left(A_{1}^{r}, B_{1}^{S-1}\right) & =\infty
\end{aligned}
$$

in which $d\left(a^{\prime}, b^{\prime}\right)$ is the distance that we set between $a^{\prime}$ and $b^{\prime}$.

We refer to the use of the value $\delta_{\lambda \text {,delA }}$ as the "delA procedure," $\delta_{\lambda, \text { delB }}$ as the "delB procedure," and $\delta_{\lambda, \text { match }}$ as the "match procedure." By allowing three means of aligning the elements, we can align elements with different time like DTW. In addition, we use $\delta_{\lambda, \text { match }}\left(A_{1}^{r}, B_{1}^{S}\right)$ and the constraint $\delta_{\lambda, \operatorname{delB}}\left(A_{1}^{r}, B_{1}^{S-1}\right)=\infty$ for treating the part whose end possibility is no more than 0.5 as an event and modifying its duration.

3.1.3 Costs for alignment procedure. We now describe the costs of the procedures.

Here, $d\left(a_{r}, b_{s}\right)$ represents the distance between aligned elements. We call "delA procedure" and "delB procedure" as "delete procedure". For "delete procedure", the value of the alignment costs is $d\left(a_{r}, b_{s}\right)$, as shown in Eqs. 7 and 8. The cost for the "match procedure" is $d\left(a_{r}, b_{s}\right)+\lambda|d n|+g(r, s)+m c$, as shown in Eqn. 6. We describe the components of this cost in the following.

$\lambda|d n|$ is the penalty for the final consecutive "delete procedure". $d n$ is the number of "delA procedures" minus the number of "delB procedures" since the last "match procedure." Based on the calculation method, $|d n|$ is the number of consecutive "delA procedures" or consecutive "delB procedures." By adding $\lambda|d n|$, we expect "match procedure" will come before "delete procedure" when aligning consecutive elements corresponding to the same event, because the penalty of the last "delete procedure" is not added when determining the alignment of the previous elements of "match procedure."

When $d n>0$,

$g(r, s)=s v\left\{-d\left(a_{r-1}, b_{s-1}\right)+d n \cdot\left(d\left(a_{r-1}, b_{s}\right)+d\left(a_{r}, b_{s+1}\right)\right)\right\}$

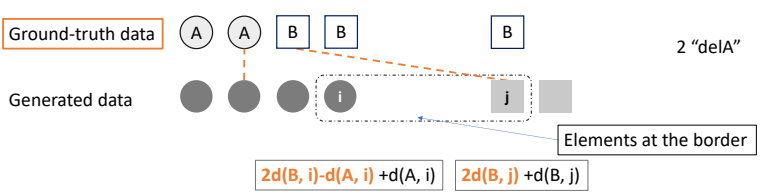

Figure 2: The orange letter shows the influence of the "delA procedure," as mentioned in Eqn. 10.

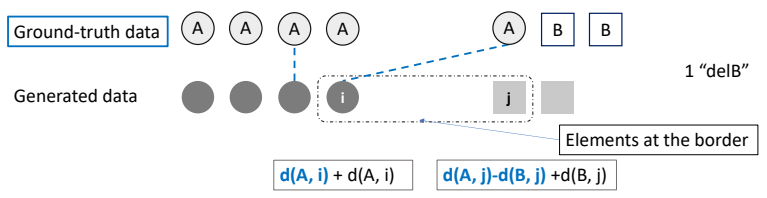

Figure 3: The blue letter shows the influence of the "delB procedure," as mentioned in Eqn. 11.

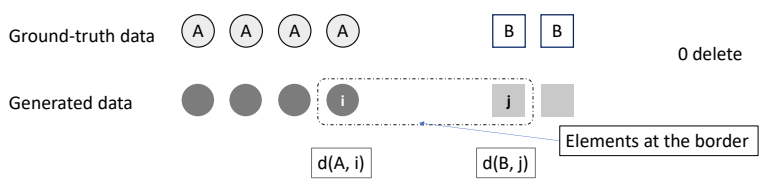

Figure 4: No "delete procedure" takes place, and $g(r, s)=\mathbf{0}$. Loss is brought about only through a "match procedure."

and when $d n<0$

$$
g(r, s)=s v\left\{-d\left(a_{r+1}, b_{s+1}\right)-d n \cdot\left(d\left(a_{r+1}, b_{s}\right)+d\left(a_{r}, b_{s-1}\right)\right)\right\} .
$$

The value of $g(r, s)$ is 0 when $d n=0$.

$g$ is set to make the duration of the corresponding parts closer. Figures 2, 3, and 4 illustrate how $g(r, s)$ operates. In these figures, we show the loss related to elements in the generated data near the event border, $i$ and $j$. The dotted line indicates "match procedure" alignment adjacent to "delete procedure". $d(\cdot, \cdot)$ is the distance function; for example, $d(A, i)$ denotes the distance between element $A$ in the GT data and element $i$ in the generated data. We show the cases in which the duration of event A is (1) longer in the generated data, (2) longer in the GT data, and (3) the same in both data. If there are GT samples in Figures 2, 3, and 4 related to the same text as the generated data, the summation of the loss is $3 d(A, i)+2 d(B, i)$ for element $i$ and $d(A, j)+4 d(B, j)$ for element $j$. In that case, the border remains at the same position. If there are only GT samples in Figures 2 and 4, the summation of the loss is $d(A, i)+2 d(B, i)$ for element $i$ and $4 d(B, j)$ for element $j$. Then, element $i$ becomes closer to $B$ than $A$, and thus the border of the events changes.

We expect the last element of certain event in the generated data to be aligned with "match procedure" just after "delB procedure" if in case (2) and the last element has a difference in value from other elements belonging to the same event because aligning surplus element in the GT data with the last element increases the cost. We think the last element rarely has a value sufficiently close to other elements in the same event in case (2) because the state is unstable.

In addition, we expect the next element of the last element for certain event in the generated data to be aligned through a "match 
procedure" just after the "delA procedure" in case (1).

$$
m c=s g\left[\min \left\{\begin{array}{l}
d\left(a_{r}, b_{s}\right) \\
d\left(a_{r-1}, b_{s-1}\right) \\
d\left(a_{r+1}, b_{s+1}\right) \\
d\left(a_{r-1}, b_{s}\right) \\
d\left(a_{r}, b_{s-1}\right)
\end{array}\right]\right.
$$

With $m c$, we expect elements with closer values to be tied together. Without $m c$, matching procedure between dissimilar elements may be prioritized because two elements are used at a distance cost of one pair of elements, whereas the "delete procedure" uses only one element. We ensure that $m c$ is no larger than $d\left(a_{r-1}, b_{s}\right)$ or $d\left(a_{r}, b_{s-1}\right)$, because otherwise, consecutive "delA procedure" and "delB procedure" may result in a smaller cost than "match procedure." Although "delA procedure" and "delB procedure" cannot be consecutive, it is undesirable.

When the first phase ends, we obtain the best alignment between the generated data at this point and the GT data. This alignment minimizes $\delta\left(A_{1}^{\text {endpos }}, B_{1}^{S}\right)$, and

$$
\delta\left(A_{1}^{p}, B_{1}^{q}\right)=\min \left\{\begin{array}{l}
\delta\left(A_{1}^{p-1}, B_{1}^{q}\right)+d\left(a_{p}, b_{q}\right)+\lambda \\
\delta\left(A_{1}^{p-1}, B_{1}^{q-1}\right)+d\left(a_{p}, b_{q}\right)+m c \\
\delta\left(A_{1}^{p}, B_{1}^{q-1}\right)+d\left(a_{p}, b_{q}\right)+\lambda
\end{array}\right.
$$

Exceptionally, $\delta\left(A_{1}^{\text {endpos-1 }}, B_{1}^{S}\right)$ and $\delta\left(A_{1}^{\text {endpos }}, B_{1}^{S-1}\right)$ are set to $\infty$ in order to align the last element of two time-series data with a "match procedure." This helps to learn the proper end position.

In the second part, element of the generated data is made closer to the aligned element of the GT data with "match procedure" or "delA procedure" in the alignment at the end of the first phase.

\subsection{DILATE-VL}

DILATE allows for the time difference between the corresponding elements of GT and generated data. Whereas DTWL-VL uses only one alignment, DILATE uses all possible alignments for training.

DILATE is only applied to time-series data with a fixed length. Therefore, we consider extending the method to generate variablelength time-series data. For this purpose, loss is added such that the generated output has the same length as the GT data, as in [21]. In this study, the authors indicate that the loss is only computed for the active part of the motion and does not include the padded part, since all sequences are padded to have an equal length owing to the implementation details. We determined the end position to be the first element whose end possibility is more than 0.5 , as in [22]. We use this method with DILATE and refer to it as DILATEVL-active. However, this may result in longer data than the average length. Consider five GT data with the same text, where the $i$ th GT data ends at the $i$ th element. When generating time-series data with the same text and a length of 10 , the end possibility becomes $1 / 5,1 / 4,1 / 3,1 / 2,1$ from the first to the fifth element. To solve this problem, we propose the addition of loss such that all of the end possibilities of the elements after the end position will be close to 1. If trained in this manner, the end possibility from the first to the last element becomes $1 / 5,2 / 5,3 / 5,4 / 5,1,1,1,1,1,1$, which will generate data with an average length. We apply this method with DILATE and refer to it as a DILATE-VL-pad.
Table 1: Duration of actions

\begin{tabular}{ccl}
\hline Action & Minimum duration & Fluctuation range \\
\hline Run & 6 & 13 \\
Walk & 7 & 21 \\
Standing & 5 & 16 \\
Sitting & 6 & 11 \\
Stand up & 10 & 0 \\
Sit down & 10 & 0 \\
Run while waving & 6 & 13 \\
Walk while waving & 7 & 21 \\
Wave & 3 & 0 \\
\hline
\end{tabular}

\section{DATASET}

To investigate performance of methods to reflect the proper order and natural duration of events stated in the input text on the generated time-series data with variable length, a dataset that provides time-series data including multiple sequential events whose duration cannot be precisely known, as well as the corresponding text, is needed. However, there are no datasets that meet our needs. Thus, we prepared a new synthetic dataset for this study.

We prepared a simple dataset to compare performance of methods easily. There are two characters in the data. The time-series data represent the actions of each character at each time as a label. There were nine types of action labels: run, walk, standing, sitting, stand up, sit down, run while waving, walk while waving, and wave.

We regard the consecutive number of times a character takes the same action as a "duration". In this dataset, the duration of each action is not constant, and the exact duration was not explained in the text. As a result, the data with the same text may differ. The duration of each action in the data was set as the summation of minimum duration and an integer selected from [0,fluctuation range] with equal probability. The value of "minimum duration" and "fluctuation range" for each action was set as indicated in Table 1. The text in the dataset have more than 40 words. We provide an example data in Figure 5.

As the content of the data was directly related to its action, this dataset could aid in determining whether the model could generate output with the proper meaning from the text.

We believe that characteristics such as variable duration of actions and relationship between the order of events and the meaning of text are common to both this dataset and real time-series data like video clips and avatar motions. For this, we expect this dataset to provide a simple task similar to video or motion generation.

\section{EXPERIMENTS}

We conducted several experiments to compare the performance of the methods. Moreover, we investigated the relationship between the output length and training time of DTWL-VL and DILATE.

\subsection{Comparison of methods}

We carried out experiments to generate variable-length time-series data from input text using several method of training, and compared their performance based on several evaluation metrics. We 


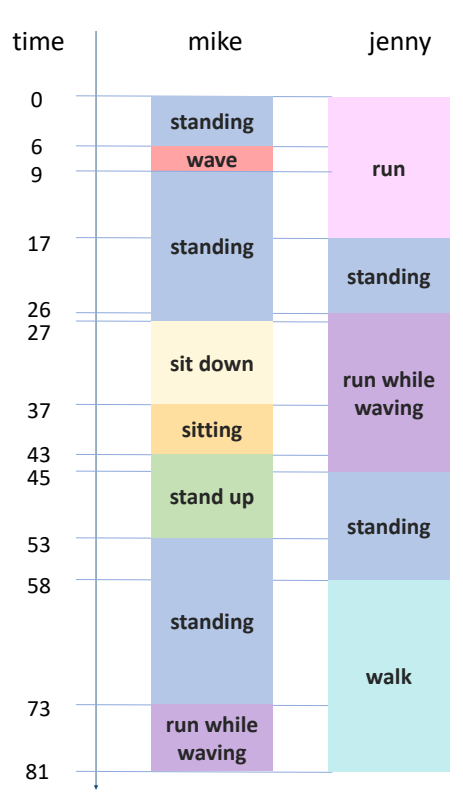

Figure 5: An example found in the dataset.The related text is "jenny was running around . then mike waved . then jenny stopped , and jenny began running while waving . mike sat down, and mike stood up . jenny stopped, and jenny began walking, and mike began running while waving ."

compared the performance of four training method, i.e., a variant of MSE, DILATE-VL-active, DILATE-VL-pad, and DTWL-VL.

The same model was used for four methods. The model get text as input and outputs the probability of each action taken by each character and the probability of being the end position for each time. In the inference phase, the first position whose end possibility became more than 0.5 was referred to as the end position.

For the text processing, we used the pretrained model of Glove[20] from [8]. The model used a bidirectional Long Short-Term Memory(LSTM) [7] to extract the sequence of features from text and used LSTM to extract the features from the previous output elements. Furthermore, the model uses a one-layer attention mechanism [23] to process the information. The model was trained using PyTorch and Tesla V100. The model was trained using the Adam optimizer[11] with a learning rate of 0.001 , exceptionally a learning rate of 0.0004 for DTWL-VL, and betas $=(0.5,0.999)$. The dataset is described in Section 4. We used 8000 data for training, 1000 data for evaluation, and 1000 data for inference. The model was trained for a maximum of 250 epochs with early stopping, exceptionally a maximum of 500 epochs with early stopping for DTWL-VL.

We represent the end possibility of an element at time i as $p_{i}^{e n d}$, the end position of the GT data as pos, and the maximum length of the generated data as maxpos. For the variants of MSE and DILATEVL-active, the loss for the end possibility $\mathcal{L}_{e}$ was

$$
\mathcal{L}_{e}=-\gamma \log \left(p_{\text {pos }}^{\text {end }}\right)-\sum_{i=1}^{\text {pos }-1} \log \left(\left(1-p_{i}^{\text {end }}\right)\right),
$$

and for the DILATE-VL-pad, the loss for the end possibility $\mathcal{L}_{e}$ was

$$
\mathcal{L}_{e}=-\sum_{i=\text { pos }}^{\text {maxpos }} \log \left(p_{i}^{\text {end }}\right)-\sum_{i=1}^{\text {pos }-1} \log \left(\left(1-p_{i}^{\text {end }}\right)\right) .
$$

$\gamma$ is a hyperparameter set to 4 aimed at alleviating imbalance in number of elements at end position and other elements, as in [13].

For the variant of MSE, DILATE-VL-active, and DILATE-VL-pad,

$$
d\left(\boldsymbol{a}^{\prime}, \boldsymbol{b}^{\prime}\right)=\frac{-\boldsymbol{b}^{\prime} \cdot\left(\log \left(\boldsymbol{a}^{\prime}\right)-\log \left(\boldsymbol{b}^{\prime}\right)\right)}{\text { num }_{\text {label }}}
$$

was used as distance between elements. Here, " . " means inner product. For GT data, action probability vector is a one-hot vector. $\boldsymbol{a}^{\prime}, \boldsymbol{b}^{\prime}$ is the concatenation of action probability vectors of all characters. num $_{\text {label }}=9$ in the experiments. Using this, the loss of generated time-series $\mathcal{L}_{s}$ was calculated. For the variants of MSE,

$$
\mathcal{L}_{s}=\frac{\sum_{i=1}^{p o s} d\left(a^{i}, b^{i}\right)}{p o s}
$$

For DILATE-VL-active and DILATE-VL-pad, $\mathcal{L}_{s}$ is calculated as in the original study. The loss for training is

$$
\alpha \cdot \mathcal{L}_{s}+\beta \cdot \mathcal{L}_{e}
$$

$\alpha, \beta=2,0.01$ for the variants of MSE and DILATE-VL-active, and 2, 0.0001 for DILATE-VL-pad.

For DTWL-VL, we defined

$$
\begin{gathered}
d\left(a^{\prime}, b^{\prime}\right)=\kappa \cdot \frac{-b^{\prime} \cdot\left(\log \left(a^{\prime}\right)-\log \left(b^{\prime}\right)\right)}{n_{\text {label }}}+\lambda \cdot\{E n d L-s g(E n d L)\} \\
E n d L=-E \log \left(p^{\text {end }}\right)-(1-E) \log \left(1-p^{\text {end }}\right)
\end{gathered}
$$

where $\kappa, \lambda=2,0.1$. Here, $E$ was 0 if the index of $b^{\prime}$ was less than the length of the GT data, and was 1 otherwise. In addition, $p^{e n d}$ is the end possibility corresponding to $a$.

We used "DTW," "MSE variant," "length difference," and "deleted ratio" as the evaluation metrics. "DTW" was the DTW value between the generated and GT data. Moreover, "MSE variant" was the average value of the distance between the elements of the generated and GT data at the same time. For "DTW" and "MSE variant," Eqn. 16 was used as the distance between elements. The deleted ratio is the ratio of deleted elements in the minimal cost alignment for DTW. Finally, "length difference" was the average of

(|GT data length - generated data length $\mid$ )/(GT data length). (21)

"DTW" can consider the time difference between corresponding events and represents the difference of aligned elements, whereas "MSE variant" cannot consider such difference. Furthermore, "deleted ratio" indicates the difference in the duration for corresponding parts based on the alignment by "DTW," whereas "length difference" represents the difference in the entire data length. Since the goal for this task was to generate data with the proper order and natural duration of events, we considered "DTW" to be a better indicator than "MSE variant" for the order of events, and "deleted ratio" to be better than "length difference" for evaluating the duration of events. In all cases, a small value indicates a better performance. The experiment was carried out twice under each setting.

In addition, we conducted an experiment to determine the relationship between the length of the data and the training time for DILATE and DTWL-VL. We use DILATE because the length of data 
Table 2: Evaluation based on DTW

\begin{tabular}{ccc}
\hline Method & DTW & Deleted ratio \\
\hline Variant of MSE & $7075.507 \pm 143.097$ & $\mathbf{0 . 1 7 2} \pm \mathbf{0 . 0 2 8}$ \\
DILATE-VL-active & $2394.312 \pm 16.487$ & $0.246 \pm 0.012$ \\
DILATE-VL-pad & $\mathbf{1 6 6 8 . 4 4 6} \pm \mathbf{2 0 . 7 8 1}$ & $0.194 \pm 0.000$ \\
DTWL-VL & $1687.914 \pm 10.263$ & $0.204 \pm 0.000$ \\
\hline
\end{tabular}

Table 3: Evaluation based on variant of MSE

\begin{tabular}{ccl}
\hline Method & MSE variant & Length difference \\
\hline variant of MSE & $\mathbf{0 . 1 3 3} \pm \mathbf{0 . 0 1 2}$ & $0.254 \pm 0.066$ \\
DILATE-VL-active & $0.363 \pm 0.066$ & $0.184 \pm 0.034$ \\
DILATE-VL-pad & $0.525 \pm 0.011$ & $\mathbf{0 . 0 8 9} \pm \mathbf{0 . 0 0 1}$ \\
DTWL-VL & $0.922 \pm 0.028$ & $0.156 \pm 0.006$ \\
\hline
\end{tabular}

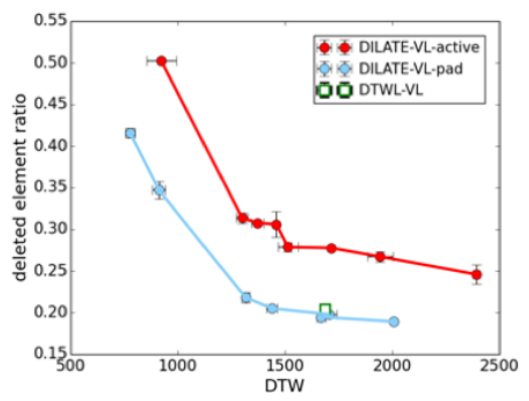

Figure 6: DTW and deleted ratio for DILATE-VL-active, DILATE-VL-pad, and DTWL-VL.

is fixed in this experiment. We only use the first phase of DTWL-VL for this experiment because it requires more time than the second phase. We used the code and data from [12] and changed the output length. We compared the training times for the same number of epochs. The experiment was conducted once under each setting.

\subsection{Results of each method and analysis}

The performance of each method is summarized below.

The MSE variant had the worst "DTW" value, indicating that a variant of MSE is unsuitable for generating time-series data including actions with the proper order. DTWL-VL performed better than DILATE-VL-active. However, DILATE-VL-pad performed better than DTWL-VL in that they exhibited no significant difference in the "DTW" value, but had a significant difference in the "deleted ratio". For "deleted ratio" DILATE-VL-pad performed better than DILATE-VL-active, as per our expectations. In DILATE-VL-active and DILATE-VL-pad, "deleted ratio" and "DTW" were affected by one hyperparameter. The two values for each method with various hyperparameter values are shown in Fig. 6.

As shown in Table 1, the duration of "wave" was short compared to the minimum duration and fluctuation range of the other actions. When the probability of "wave" was the highest among all actions for the corresponding character, we regarded the character as conducting "wave" action at that time. To verify whether the model
Table 4: Number of generated "wave" actions (2133 in GT)

\begin{tabular}{cc}
\hline Method & "Wave" number \\
\hline Variant of MSE & $0 \pm 0.00$ \\
DILATE-VL-active & $662.5 \pm 125.16$ \\
DILATE-VL-pad & $1715 \pm 57.98$ \\
DTWL-VL & $\mathbf{1 8 7 0} \pm \mathbf{2 4 . 0 4}$ \\
\hline
\end{tabular}

Table 5: Training time in relation to output length

\begin{tabular}{ccl}
\hline Output length & DTWL-VL & DILATE \\
\hline 20 & 614.19 & 1290.71 \\
50 & 1131.03 & 2345.54 \\
100 & 2306.18 & 5227.06 \\
150 & 3437.85 & 14302.21 \\
200 & 4851.29 & 40473.02 \\
250 & 5580.46 & 49982.86 \\
\hline
\end{tabular}

could output short events, we counted the number of times "wave" action was conducted. The results are presented in Table 4 . The variant of MSE could not generate "wave" action, indicating that methods such as MSE have difficulty in reflecting events with a short duration, and consequently cannot reflect the order of events properly. Therefore, we infer that the method lacks the capacity to reflect short events in video or motion generation tasks.

The results of the training time related to the output length are presented in Table 5. DTWL-VL required less time, and the difference in training time increased as the output length increased.

The experiments revealed that, although the DILATE-VL-pad outperformed DTWL-VL, DTWL-VL required less time for training and may be suitable for generating long time-series data.

\section{CONCLUSIONS}

We proposed DTWL-VL for reflecting the correct order of events stated in an input text and proper duration of such events in the output time-series data. We demonstrated that DTWL-VL and DILATEbased methods, which can make the generated data and GT data closer by considering the alignments, are superior to a variant of MSE for generating data with a proper order of events. Moreover, the calculation of the end possibility for the padded part of the sequence results in a better performance when using DILATE for the generation of variable-length time-series data.

Although DILATE-VL-pad achieved a higher performance than DTWL-VL, DTWL-VL required less training time, which may be an advantage when generating long time-series data. We expect that consideration of alignment between time-series data like DTWL-VL or DILATE improves fidelity of data for generating motion or video sequences from text.

\section{ACKNOWLEDGMENTS}

We would like to thank Kohei Uehara and Naoyuki Gunji for helpful advice. This work was supported by JSPS KAKENHI Grant Number JP19H01115, JP20H05556, and Moonshot R\&D Grant Number JPMJPS2011. 


\section{REFERENCES}

[1] Hyemin Ahn, Timothy Ha, Yunho Choi, Hwiyeon Yoo, and Songhwai Oh. 2018 Text2Action: Generative Adversarial Synthesis from Language to Action. In IEEE International Conference on Robotics and Automation. 5915-5920. https: //doi.org/10.1109/ICRA.2018.8460608

[2] Yogesh Balaji, Martin Renqiang Min, Bing Bai, Rama Chellappa, and Hans Peter Graf. 2019. Conditional GAN with Discriminative Filter Generation for Text-toVideo Synthesis. In Proceedings of the Twenty-Eighth International foint Conference on Artificial Intelligence. International Joint Conferences on Artificial Intelligence Organization, 1995-2001. https://doi.org/10.24963/ijcai.2019/276

[3] Qi Chen, Qi Wu, Jian Chen, Qingyao Wu, Anton van den Hengel, and Mingkui Tan. 2020. Scripted Video Generation With a Bottom-Up Generative Adversarial Network. IEEE Transactions on Image Processing 29 (2020), 7454-7467. https: //doi.org/10.1109/TIP.2020.3003227

[4] Marco Cuturi and Mathieu Blondel. 2017. Soft-DTW: A Differentiable Loss Function for Time-Series. In Proceedings of the 34th International Conference on Machine Learning - Volume 70.

[5] Kangle Deng, Tianyi Fei, Xin Huang 0006, and Yuxin Peng. 2019. IRC-GAN: Introspective Recurrent Convolutional GAN for Text-to-video Generation. In Proceedings of the Twenty-Eighth International foint Conference on Artificial Intelligence. 2216-2222. https://doi.org/10.24963/ijcai.2019/307

[6] Sakoe Hiroaki and Chiba Seibi. 1978. Dynamic programming algorithm optimization for spoken word recognition. IEEE Transactions on Acoustics, Speech, and Signal Processing 26, 1 (1978), 43-49. https://doi.org/10.1109/TASSP.1978.1163055

[7] Sepp Hochreiter and Jürgen Schmidhuber. 1997. Long Short-term Memory. Neural computation 9 (1997), 1735-80. https://doi.org/10.1162/neco.1997.9.8.1735

[8] Jeffrey Pennington and Richard Socher and Christopher D. Manning. 2014. GloVe Global Vectors for Word Representation. https://nlp.stanford.edu/data/glove.6B.zip

[9] Doyeon Kim, Donggyu Joo, and Junmo Kim. 2020. TiVGAN: Text to Image to Video Generation With Step-by-Step Evolutionary Generator. IEEE Access 8 (2020), 153113-153122. https://doi.org/10.1109/ACCESS.2020.3017881

[10] Jaehyeon Kim, Sungwon Kim, Jungil Kong, and Sungroh Yoon. 2020. Glow-TTS: A Generative Flow for Text-to-Speech via Monotonic Alignment Search. In Advances in Neural Information Processing Systems. 8067-8077. https://proceedings. neurips.cc/paper/2020/file/5c3b99e8f92532e5ad1556e53ceea00c-Paper.pdf

[11] Diederik Kingma and Jimmy Ba. 2014. Adam: A Method for Stochastic Optimization. International Conference on Learning Representations (2014).

[12] Vincent LE GUEN and Nicolas THOME. 2019. Shape and Time Distortion Loss for Training Deep Time Series Forecasting Models. In Advances in Neural Information Processing Systems. https://proceedings.neurips.cc/paper/2019/file/ 466accbac9a66b805ba50e42ad715740-Paper.pdf

[13] Naihan Li, Shujie Liu, Yanqing Liu, Sheng Zhao, and Ming Liu. 2019. Neural Speech Synthesis with Transformer Network. Proceedings of the AAAI Conference on Artificial Intelligence 33 (2019), 6706-6713. https://doi.org/10.1609/aaai.v33i01. 33016706

[14] Yitong Li, Martin Min, Dinghan Shen, David Carlson, and Lawrence Carin. 2018 Video Generation From Text. https://aaai.org/ocs/index.php/AAAI/AAAI18/ paper/view/16152

[15] Angela S. Lin, Wu Lemeng, Corona Rodolfo, Tai Kevin, Huang Oixing, and Raymond J. Mooney. 2018. Generating Animated Videos of Human Activities from Natural Language Descriptions. In Proceedings of the Visually Grounded Interaction and Language Workshop at NeurIPS. http://www.cs.utexas.edu/users/ai-labpubview.php?PubID=127730

[16] Yue Liu, Xin Wang, Yitian Yuan, and Wenwu Zhu. 2019. Cross-Modal Dual Learning for Sentence-to-Video Generation. In Proceedings of the 27th ACM International Conference on Multimedia (Nice, France) (MM '19). Association for Computing Machinery, New York, NY, USA, 1239-1247. https://doi.org/10.1145/ 3343031.3350986

[17] Tanya Marwah, Gaurav Mittal, and Vineeth N. Balasubramanian. 2017. Attentive Semantic Video Generation Using Captions. In 2017 IEEE International Conference on Computer Vision. https://doi.org/10.1109/ICCV.2017.159

[18] Gaurav Mittal, Tanya Marwah, and Vineeth N. Balasubramanian. 2017. SyncDRAW: Automatic Video Generation Using Deep Recurrent Attentive Architectures. In Proceedings of the 25th ACM International Conference on Multimedia. https://doi.org/10.1145/3123266.3123309

[19] Yingwei Pan, Zhaofan Qiu, Ting Yao, Houqiang Li, and Tao Mei. 2017. To Create What You Tell: Generating Videos from Captions. In Proceedings of the 25th ACM International Conference on Multimedia. https://doi.org/10.1145/3123266.3127905

[20] Jeffrey Pennington, Richard Socher, and Christopher Manning. 2014. GloVe Global Vectors for Word Representation. In Proceedings of the 2014 Conference on Empirical Methods in Natural Language Processing. Association for Computational Linguistics, Doha, Qatar, 1532-1543. https://doi.org/10.3115/v1/D14-1162

[21] Matthias Plappert, Christian Mandery, and Tamim Asfour. 2018. Learning a bidirectional mapping between human whole-body motion and natural language using deep recurrent neural networks. Robotics and Autonomous Systems 109 (2018), 13 - 26. https://doi.org/10.1016/j.robot.2018.07.006
[22] Jonathan Shen, Ruoming Pang, Ron J. Weiss, Mike Schuster, Navdeep Jaitly, Zongheng Yang, Zhifeng Chen, Yu Zhang, Yuxuan Wang, Rj Skerrv-Ryan, Rif A. Saurous, Yannis Agiomvrgiannakis, and Yonghui Wu. 2018. Natural TTS Synthesis by Conditioning Wavenet on MEL Spectrogram Predictions. In IEEE International Conference on Acoustics, Speech and Signal Processing. 4779-4783. https://doi.org/10.1109/ICASSP.2018.8461368

[23] Ashish Vaswani, Noam Shazeer, Niki Parmar, Jakob Uszkoreit, Llion Jones, Aidan N Gomez, $€$ ukasz Kaiser, and Illia Polosukhin. 2017. Attention is All you Need. In Advances in Neural Information Processing Systems. https://proceedings. neurips.cc/paper/2017/file/3f5ee243547dee91fbd053c1c4a845aa-Paper.pdf

[24] Nguyen Tan Viet Tuyen, Armagan Elibol, and Nak Young Chong. 2020. Learning from Humans to Generate Communicative Gestures for Social Robots. In 2020 17th International Conference on Ubiquitous Robots. https://doi.org/10.1109/ UR49135.2020.9144985

[25] Tatsuro Yamada, Hiroyuki Matsunaga, and Tetsuya Ogata. 2018. Paired Recurrent Autoencoders for Bidirectional Translation Between Robot Actions and Linguistic Descriptions. IEEE Robotics and Automation Letters 3, 4 (2018), 3441-3448. https: //doi.org/10.1109/LRA.2018.2852838 